\title{
Mark Mulligan leads Emory swine flu vaccine trial
}

Fe bination with the return of children to school could trigger a spike in swine flu cases, the National Institute of Allergy and Infectious Diseases has begun clinical trials of candidate vaccines against the pandemic influenza (H1N1) virus. The JCI spoke with Mark Mulligan (Figure 1), professor of medicine at Emory University School of Medicine and principal investigator at the Emory Vaccine Center's Vaccine and Treatment Evaluation Unit (VTEU), one of eight in a nationwide network of VTEUs where the trials will be conducted.

JCI: The Emory Vaccine Center is experienced in developing candidate vaccines for HIV, malaria, and H5N1 avian influenza. So is this business as usual for your team?

Mulligan: No, not by any means. These are extraordinary circumstances. We are in the midst of a public health emergency, and the sense of urgency to control this pandemic is greater than anything I've experienced in my career.

JCI: What are the primary objectives of this trial?

Mulligan: We hope to determine the strength $(15 \mu \mathrm{g}$ vs. $30 \mu \mathrm{g})$ and number of doses (one vs. two) of the vaccine required to stimulate an immune response likely to protect against $\mathrm{H} 1 \mathrm{~N} 1 \mathrm{flu}$. We will evaluate vaccine safety and immunogenicity in five different age groups: 6 to 35 months, 3 to 9 years, 10 to 17 years, 18 to 64 years, and adults 65 years and older. We also hope to determine how best to give the H1N1 vaccine safely in combination with the seasonal flu vaccine and without reducing the immune response to either.

JCI: Do you anticipate children, adults, and seniors responding differently to the vaccine?

Mulligan: The influence of age has been very interesting in this pandemic. The burden of disease has been in children and young adults, while seniors have been mostly spared. Seniors may have had previous exposure to an H1N1-like virus that primed their immunological memory, providing a level of protection against H1N1. This could influence their response to vaccine; they may only require one dose. If true, this could be very important during the early stages of vaccine deployment if supply is limited.

JCI: What have been some of the chal- lenges in producing this vaccine?

Mulligan: Influenza is unpredictable, and there are always difficulties with the manufacturing process. The efficiency with which the vaccine strain grows in embryonated hens' eggs has only been about 30\% of what is considered optimal. Over time that rate often improves; however, we don't have the luxury of time on this occasion.

JCI: When is a vaccine estimated to be available?

Mulligan: Eighty million doses are projected to be available in September and 120 million in October. The vaccine will be held by the federal government and deployed by state governments depending on when it is needed.

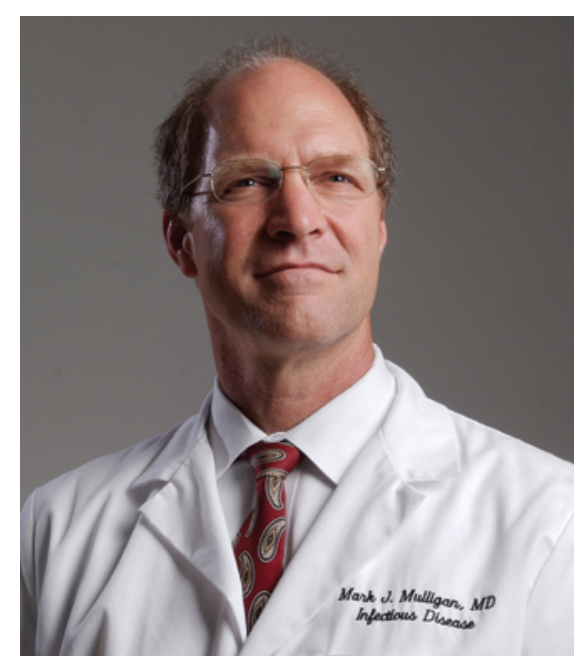

Figure 1

Mark Mulligan says that the sense of urgency to control the current $\mathrm{H} 1 \mathrm{~N} 1$ swine flu pandemic is greater than anything he has experienced in his career.

JCI: What members of the population would first receive the vaccine?

Mulligan: The Advisory Committee on Immunization Practices has recommended that five high-priority groups receive the vaccine: pregnant women, children and young adults 6 months to 24 years of age, household contacts of children younger than 6 months, health-care workers and emergency health-care responders, and nonelderly adults with underlying medical conditions that increase their risk of complications from influenza. If this virus changes, these recommendations may change; however, there is currently no evidence to suggest that the virus is mutating.

JCI: Are there plans to add the H1N1 component to the seasonal flu vaccine?

Mulligan: Two or three injections (one for seasonal flu, one or two for swine flu) will be required. Going forward, if there is a need to include the $\mathrm{H} 1 \mathrm{~N} 1$ vaccine in the seasonal flu vaccine, it will likely be feasible to develop a quadrivalent vaccine.

JCI: The USA only makes about $20 \%$ of its seasonal flu vaccine demand. In light of the global nature of the $\mathrm{H} 1 \mathrm{~N} 1$ pandemic, are there concerns some countries may limit vaccine export?

Mulligan: This is a global phenomenon, and the virus does not respect national borders. But in modern political reality, this is an important question for governments and policy makers to consider. It will be key to increase our own production capacity as well as develop more modern technology for flu vaccine production, such as cell culture-based systems.

JCI: Has the H1N1 pandemic given momentum to the proposal of a widespread prepandemic vaccination strategy for H5N1 avian flu?

Mulligan: For the past ten years, we have really raised our awareness and level of preparedness for a pandemic of influenza of whatever origin, and this has been very beneficial in terms of our response to swine flu. We were expecting a pandemic of avian flu, and so it is interesting that it has turned out to be swine flu. We shouldn't be surprised - these are unpredictable viruses. The focus right now is not on H5N1, and appropriately so.

JCI: Frequent hand washing and limited hand-to-hand contact are encouraged to minimize disease spread. Do you greet all your visitors with a squirt of hand sanitizer?

Mulligan: I have to confess that I am not doing that! Those kinds of efforts, along with staying home from school or work when ill, are very worthwhile. I have, however, learned to cough in the "new" way - covering my mouth with the crook of my arm instead of the palm of my hand. Maybe I do need to get a bottle of hand sanitizer.

\section{Brooke Grindlinger}

